Instituto Internacional de Investigación y Desarrollo Tecnológico Educativo INDTEC, C.A.

DOI: https://doi.org/10.29394/scientific.issn.2542-2987.2016.1.1.10.169-189

OAI-PMH: http://www.indteca.com/ojs/index.php/Revista Scientific/oai

\title{
Reutilización de Residuos Sólidos como Alternativa de Formación en la Conservación del Ambiente Elaborando Nuevos Materiales para el Docente de Educación Inicial
}

\author{
Autora: Francys Yuviana Garrido Rojas \\ Universidad Pedagógica Experimental Libertador, UPEL \\ enyuby@hotmail.com \\ Barinas, Venezuela
}

\section{Resumen}

El estudio enfoca su objetivo en establecer la reutilización de residuos sólidos como alternativa de formación en la conservación del ambiente elaborando nuevos materiales para los docentes del C.E.I.N. "Sebastián Araujo Briceño" de Ciudad Bolivia, municipio Pedraza estado Barinas. La naturaleza de la investigación es cualitativa, el método es la Investigación Acción. Los informantes claves estarán constituidos por la directora de la institución, la coordinadora Pedagógica y una docente de aula. La técnica empleada será la entrevista semiestructurada, y el instrumento la guía de entrevista. El análisis de la información se realizará mediante la codificación, categorización, triangulación y estructuración de teorías. En conclusión, a través de la experiencia que ha tenido la investigadora en esta institución, se ha podido constatar que existe una necesidad imperante en cuanto a la formación del personal de la institución en relación a la importancia y beneficios de la conservación del ambiente y del mismo modo, la necesidad de contar con materiales didácticos y pedagógicos que acompañen los diferentes espacios donde se atienden a los niños, toda esta realidad, le da un sentido importante al presente estudio. 


\title{
Reuse of Solid Waste as Alternative Training Environment Conservation for Brewing in New Materials of Initial Education Teacher
}

\begin{abstract}
The study focuses its objective to establish the reuse of solid waste as an alternative training in environmental conservation by developing new materials for teachers C.E.I.N. "Sebastian Araujo Briceño" Ciudad Bolivia, Barinas Pedraza municipality. The nature of the research is qualitative, the method is Action Research. Key informants shall consist of the director of the institution, the educational coordinator and classroom teacher. The technique used is the semi-structured interview, and the interview guide instrument. The analysis of information will be done through coding, categorization, triangulation and structuring theories. In conclusion, through the experience that has been the research in this institution, it has been shown that there is a pressing need in terms of training of staff of the institution in relation to the importance and benefits of environmental conservation and Similarly, the need for teaching and learning materials accompanying the different spaces where children are served, all this reality, gives an important sense this study.
\end{abstract}

Keywords: solid waste; environmental conservation; materials. 


\section{Introducción}

Con las nuevas perspectivas que abren el progreso científico e industrial en las sociedades modernas, la consecuente pérdida del sentido de la vida en sectores cada vez más amplios de la población, la fragmentación del equilibrio natural, así como también, la constante denuncia de la degradación natural, son características de nuestro mundo, que no por casualidad tienden a asociarse cada vez más, surgiendo de esta manera nuevas e inquietantes formas de atentar contra la naturaleza y los elementos que la conforman.

Es por ello, que se vienen realizando estudios en cuanto a los impactos ocasionados por el hombre a la naturaleza que ha permitido determinar la apremiante necesidad de realizar aportes que vayan directamente a cambiar la actuación del hombre en función de conservar el ambiente. Una de las alternativas que ha venido sumando fuerza en la conservación del ambiente, ha sido el hecho concreto de profundizar en las bondades que ofrece la reutilización de gran cantidad de desechos producidos por la humanidad. En este sentido, Osorio (2012) manifiesta que:

En la actualidad la reutilización es y debe entenderse como una estrategia de aprendizaje utilizada por el hombre en la reducción del volumen de desperdicios y residuos sólidos; este proceso consiste en un conjunto de acciones que realiza el hombre sobre diferentes materiales para transformarlos y volverlos a recuperar (pág. 84).

De ahí que el reutilizar materiales (residuos sólidos) se ha convertido en una de las más efectivas estrategias para trabajar el tema de la conservación y preservación del ambiente, sobre todo entendiendo que éste ha sido altamente vulnerado ya tacado por la mano del hombre, que sin entender la importancia que el mismo reviste para mantener la vida sobre el planeta, lo ataca y lo destruye sin piedad, pensando casi siempre en lo beneficios económico y de confort que le puede generar su explotación y dejando totalmente de lado su importancia para mantener la vida en el planeta. 
Por su parte, para González (2002), hablar de enseñanza conservacionista, es enfocar un tema de gran complejidad en el mundo de la educación ambiental, pues:

Existe un notorio desatino entre lo que se enseña en relación a la educación ambiental y lo que se ve reflejado en los seres humanos a través de una práctica concreta del cuido a la diversidad de la vida en sus expresiones y manifestaciones en el planeta (pág. 83).

No podría existir una enseñanza sólida y un aprendizaje efectivo hacia la conservación del ambiente y los recursos que lo conforman, si primero los seres humanos no se forman, es decir, no interiorizan, la imperante necesidad que existe de conservar el medio que nos rodea, con todas sus expresiones de vida, para que de esta forma se pueda cuidar y preservar el espacio vital.

Sin embargo, hay sociedades y organismos a nivel mundial que cada día se han ido sumando a la consiente necesidad de activar mecanismos que vayan en pro de proteger el ambiente. De esta manera, Carvajal (2012), manifiesta que:

Ciudades como Bucaramanga en Colombia, han ido impulsando desde el sistema educativo y formación social diferentes programas que han contribuido de manera exitosa en la conservación y preservación del ambiente, dándole un uso comercial a los diferentes desechos, según su clasificación (pág. 107).

Estas alternativas se han ido convirtiendo en ejemplo para el país y para el mundo, que sigue insistiendo a través de diferentes medios y programas, la necesidad de cambiar de actitud hacia el ambiente, a través de acciones concretas que permitan contrarrestar la actitud del hombre hacia el medio que lo circunda y le proporciona todos los elementos para mantener la vida en el planeta. 
Asimismo, otros países de Latinoamérica se han ido sumando a la idea de darle una importante utilización a los diferentes desechos que día a día se producen por cientos y miles de toneladas, contribuyendo a reducir los niveles de contaminación del medio ambiente. Al respecto Zuben (2014) afirma que "Latinoamérica tienen un gran potencial en cuanto a la explotación del reciclaje pues anualmente produce unas treinta mil toneladas de desechos que pueden ser reutilizados" (pág. 7).

Esto ha generado la creciente incentivación de algunos países de la región a interesarse en el conocimiento de las bondades de los proyectos de reciclaje y las alternativas ambientales y económicas que este representa para el país. Entendiendo que la principal acción que se debe emprender, está en la formación de la ciudadanía en relación a esta alternativa, y esta formación indudablemente ha de ser impulsada desde los diferentes centros escolares, pues es a través de la educación que se logra sensibilizar y enfocar una sociedad hacia alternativas tan dinámicas e importantes como esta.

Por su parte, Venezuela ha venido sumando esfuerzos a la conservación del ambiente, desde los postulados presentes en la carta magna y las leyes que se han creado para salvaguardar los espacios naturales de la región, hasta sumarse como voz ante el mundo para apoyar los acuerdos internacionales en materia de conservación y preservación del ambiente, sin embargo, Ramírez (2013), manifiesta que "En Venezuela han venido creciendo los focos de contaminación ambiental, cada día las ciudades presentan un panorama más deplorable, que habla de la escasa conciencia ciudadana en torno al medio ambiente" (pág. 82).

En este sentido, la responsabilidad que tiene el sistema educativo en formar a la ciudadanía en torno a la conservación del ambiente es sumamente importante; pero no se podría formar a la ciudanía si antes no se forma el docente quien es el primero que está llamado a mantener una actitud cónsona. Por ello, Rodríguez (2014), afirma que "el educador es el primero que debe 
formarse en conciencia en relación a lo que va a enseñar, pues debe enseñar con la palabra y con el ejemplo" (pág. 19).

De ahí que, los educadores venezolanos son los primeros en instruirse en relación a la materia ambiental, para poder impulsar programas ambientales que reivindiquen verdaderamente los problemas ambientales presentes en la sociedad actual. Por ello, Gómez (2014), manifiesta que:

El reciclaje es una buena alternativa para disminuir los focos de contaminación en las ciudades, pero la formación de su importancia debe ser masiva y empezar por los centros educativos, mostrando las bondades que ofrece esta estrategia ambientalista y económica (pág. 58).

Todos estos planteamientos han fortalecido la idea de desarrollo de la presente investigación enfocada en reutilizar algunos residuos sólidos como alternativa de formación en la conservación del ambiente en docentes del C.E.I.N. "Sebastián Araujo Briceño" de Ciudad Bolivia, Municipio Pedraza Estado Barinas. Ya que a través de conversaciones sostenidas con algunos docentes de la institución y la observación directa en el lugar objeto de estudio se ha podido verificar que los docentes de la institución adolecen de una verdadera formación en torno a los benéficos que puede ofrecer el reutilizar residuos sólidos transformándolos en nuevos materiales para la conservación del ambiente.

Algunas de las causas que sustentan esta realdad se enmarcan en el hecho concreto de que algunos docentes no han tenido la oportunidad de profundizar en las bondades que ofrece reutilizar un material como alternativa para conservar el ambiente, conociendo solo lo elemental de esta temática, otro factor, se visualiza en la escaza o nula formación interna por parte de los coordinadores de formación docente, limitándose a formar solo los temas concernientes a planificación y evaluación de los contenidos. 
Toda esta realidad ha traído como consecuencia que los docentes se limiten a formar escasamente lo elemental y conceptual de estas temáticas a los niños y niñas no viéndose reflejada en el ambiente de la institución una cultura verdaderamente conservacionista a través de la activación de algún programa de reutilización, tomando en cuenta la comunidad, los niños y niñas que día a día asisten al recinto escolar, viéndose afectada la calidad educativa en torno a este tema, lo que ha traído como consecuencia el establecimiento de un objetivo central que se enmarca en establecer la reutilización de residuos sólidos como alternativa de formación en la conservación del ambiente elaborando nuevos materiales para los docentes del C.E.I.N. "Sebastián Araujo Briceño" de Ciudad Bolivia, municipio Pedraza estado Barinas.

\section{Objetivos General}

Establecer la reutilización de residuos sólidos como alternativa de formación en la conservación del ambiente elaborando nuevos materiales para los docentes del C.E.I.N. "Sebastián Araujo Briceño" de Ciudad Bolivia, municipio Pedraza estado Barinas

\section{Teoría y Conceptos}

Según Claret (2012), con respecto a las bases teóricas expresa que éstas "representan aquellos enfoques o corrientes desarrolladas por autores sobre el tema tratado en la investigación" (pág. 20). La cual implica, un desarrollo amplio de los conceptos que conforman el enfoque o el punto de vista que se adopta para explicar el problema enfocado. De esta manera, se desarrollan los siguientes planteamientos. 


\subsection{Reutilización de materiales}

Una de las alternativas para solucionar el problema de la contaminación es por medio de la reutilización de materiales, que a juicio de Sanabria (2012) es:

El proceso en el cual los residuos o materiales de desperdicio son recolectados y transformados en nuevos materiales que pueden ser utilizados o vendidos como nuevos productos 0 materias primas. Como por ejemplo el papel, el cartón, el vidrio, y los metales, entre otros (pág. 72).

De esta manera, la reutilización de materiales es un proceso que consta de las siguientes etapas: separar los componentes de la basura en orgánicos e inorgánicos. Clasificar los componentes inorgánicos en papel, cartón, vidrio y metales. Llevar todos estos materiales a las industrias correspondientes que los reciclan. Procesar cada material de desecho con un tratamiento adecuado.

De ahí que, en la vida cotidiana se utilizan gran cantidad de recursos naturales y al hacerlo producimos volúmenes de desechos que al ser manejados inadecuadamente ocasiona graves problemas de contaminación al ambiente. Cuando se recuperan materiales que formaron parte de la basura para darle algún uso, se están aprovechando los recursos. El reciclaje se incorpora en la sociedad como una alternativa de protección ambiental con el propósito de disminuir el flujo de residuos sólidos de origen doméstico, comercial entre otros.

\subsection{Importancia de la reutilización de materiales}

La reutilización de materiales resulta de gran importancia, pues a través de este accionar los materiales de desperdicios son recolectados y transformados en nuevos materiales que pueden ser utilizados o vendidos como nuevos productos. Entendiendo entonces que la reutilización de materiales es un proceso simple que ayuda a resolver muchos de los 
problemas creados por la forma de vida moderna que llevamos. La reutilización de materiales como técnica para la reducción de desechos, representa una de las estrategias de desarrollo más importante que ha surgido del movimiento ambiental en los últimos años.

Asimismo, el Ministerio del Poder Popular para el Ambiente (2009), define la reutilización de materiales como:

Un proceso que consiste en aprovechar materiales u objetos que la sociedad de consumo ha descartado por considéralos inútiles, es decir, darle un nuevo valor a lo descartado a fin de que se pueda utilizar en la fabricación o preparación de nuevos productos, que no tienen por qué parecerse ni en forma ni aplicación al producto original (pág. 4).

A partir de lo descrito anteriormente se puede decir que reciclar es la acción de convertir un material desechado en nuevos productos. Dentro de la importancia de la reutilización de materiales, destaca:

\subsection{Conservación del ambiente}

La concepción del medio ambiente ha estado centrada exclusivamente en el componente natural y en la consideración de las personas como centro de ese componente al cual también se denomina antropocentrismo. En el Currículo Básico Nacional (CBN, 2007), es concebido como "un todo formado por la naturaleza, el hombre, la cultura y componentes de tipo neohistóricos, económicos, políticos y culturales”. Es decir, engloba el fortalecimiento de los valores hacia el ambiente desde un punto ético y estético, promoviendo a su vez la participación ciudadana en la solución de problemas socio ambienta. Todo lo expresado, permite inferir que en las instituciones educativas se exige una educación adecuada al educando, que contribuya a crear conciencia y fomentar actitudes favorables para atenuar los graves problemas existentes en el ambiente. 
En gran parte de la crisis ambiental se debe al modelo de desarrollo que se ha seguido, el cual ha trasformado en el ambiente e incluso lo ha llevado a estados críticos, tales como el agotamiento de los recursos energéticos y alimentarios, contaminación, ruptura del equilibrio, extinción de especies, pobreza, desnutrición, consumismo, entre otros. Situación está, provocada por la acción del hombre, pues se puede inferir que aún no ha comprendido que es parte de la naturaleza y al destruirla se provoca su propia desaparición.

Para García y otros (2012), el medio ambiente es "un conjunto de factores que actual sobre un sistema y determinan el curso y forma de existencia, por ello, se puede considerar como un conjunto de métodos naturales, socioculturales y tecnológicos que interactúan entre sí, en un espacio y tiempo determinado" (pág. 29).

Entonces, en el sistema natural interactúan los animales, el agua, las plantas, el aire y el suelo, incluyendo a los seres humanos. De igual manera, el sistema socio cultural está compuesto por la familia, las instituciones orientadas para gestionar las relaciones de la comunidad con los otros sistemas, tales como: Consejos Comunales, ministerios, iglesias, hospitales, entre otros.

\subsection{Formación Conservacionista}

La formación conservacionista como elemento de cambio, puede convertirse en una herramienta transformadora de la problemática ambiental, entendiéndose ésta como todos los elementos contaminantes del medio físico, técnico y social, comprendidos en el ámbito natural, social y humano. Según González (2003), viene a constituirse en "el sendero para la fundamentación de una ética ecológica desde la perspectiva de la ética biométrica" (pág. 96), la cual recupera el valor intrínseco que tiene la naturaleza como potenciadora y generadora de vida, rompiendo con la posición de dominio del ser humano sobre la naturaleza. 
En esta orientación, la corriente ecologista ha estado muy asociada al conservacionismo, que postula el resguardo del ambiente en condiciones de no intervención humana. De allí el tecnocentrismo o ecotecnicismo que surge bajo la creencia de la superación de la crisis ambiental a través de la ciencia y la tecnología; por su parte, el humanismo crítico social emerge para instaurar un cambio radical en el ambientalismo moderno para atender las necesidades y carencias de la gente. Esta última corriente según Pardo (2003), está referida a la dualidad ambiente sociedad, caracterizada por la relación dominante del ser humano sobre la naturaleza, donde se evidencia que la asociación entre la ciencia y la tecnología desarrollan instrumentos para su trasformación, que no ha sido positiva en muchos casos.

\section{Metodología}

De acuerdo a los objetivos planteados el presente estudio se enmarca en el enfoque cualitativo porque tal como lo expresa Martínez (2004), la investigación trata de aproximarse a la realidad concreta del problema con miras a su transformación y las aplicaciones para la solución, por lo que "destaca el registro objetivo de los hechos en el momento de sucederse, generando acciones determinantes con el grupo social en estudio" (pág. 84).

Por otra parte, Murcia, (citado en Hurtado y Toro 2011), afirma que en el enfoque cualitativo "el investigador se inserta dentro de la realidad social de los grupos y comunidades para contribuir con la interpretación objetiva de la misma y buscar junto con ellos, la formulación de acciones para transformarlas" (pág. 53). Indicando con ello, que el presente estudio está dentro de esta línea del paradigma cualitativo.

\subsection{Tipo de Estudio}

Es importante destacar que en el presente estudio el tipo de investigación está enfocada en el paradigma de investigación acción, la cual 
se desarrollará en el C.E.I.N. "Sebastián Araujo Briceño" de Ciudad Bolivia municipio Pedraza, estado Barinas, buscando establecer el reciclaje como alternativa de formación en la conservación del medio ambiente en docentes del C.E.I.N. "Sebastián Araujo Briceño" de Ciudad Bolivia, municipio Pedraza estado Barinas. Se utilizará como apoyo la fenomenología, la cual parte de elementos fundamentales para dar garantía al cumplimiento de los objetivos planteados por la investigadora y dar solución al problema utilizando los métodos necesarios para el estudio y análisis de la realidad que aquejan al sector escolar.

Al respecto Martínez (2004) expresa que:

La investigación- acción realiza simultáneamente la expansión del conocimiento científico y la solución de un problema, mientras aumenta, igualmente, la competencia de sus respectivos participantes (sujetos coinvestigadores) al ser llevada a cabo en colaboración, en una situación concreta y usando la realimentación de la información en un proceso clínico. (pág. 239).

La aplicación de la investigación acción permitirá dar solución a la problemática presente en la institución, esto generará una serie de pasos para llevar a cabo los objetivos planteados, a tal fin se ejecutarán las diversas acciones para lograr los objetivos planteados. La investigación acción consistirá en establecer soluciones a las problemáticas existentes y así realizar una serie de operaciones para atacar de raíz la situación y de esta manera se dé solución paso a paso tomando en cuenta las debilidades y fortalezas que presenta el entorno escolar afectado.

\subsection{Diseño de la investigación}

Siendo el estudio de tipo investigación acción participativa, el diseño se basará en lo fenomenológico el cual se procesará la información de forma veraz y subjetiva referente al grupo en estudio. Según Paz (2011): "La 
fenomenología es una corriente de pensamiento propia de la investigación interpretativa" (pág. 63). De esta manera, la misma aportará como base del conocimiento la experiencia subjetiva inmediata de los hechos tal como se perciban.

Lo que significa que se realizará un estudio minucioso al grupo en estudio con el fin de poder interpretar subjetivamente los hechos que allí se presentan y para ello se llevará a cabo la recolección y la interpretación de los datos obtenidos durante la observación y la aplicación de la entrevista en profundidad, para luego encontrar soluciones a las necesidades y requerimientos del caso en estudio.

En el mismo orden de ideas, se precisa que el marco de la investigación cualitativa se caracteriza su adecuación y permanencia de la realidad educativa. En este caso, la realidad que viven los docentes del C.E.I.N. "Sebastián Araujo Briceño" de Ciudad Bolivia, municipio Pedraza estado Barinas. Cabe resaltar que el diseño de la investigación se llevó a cabo en las siguientes fases:

4.2.1. Fase 1. Diagnóstico. Consiste en identificar los informantes, en el grupo de docentes del C.E.I.N. "Sebastián Araujo Briceño" de Ciudad Bolivia, municipio Pedraza estado Barinas, con la finalidad de señalar las diferentes cualidades necesarias para la investigación, por otra parte, se usará la observación directa en el grupo de estudio el cual establece el enfoque cualitativo.

Según Martínez (2004). “...Para ello, el investigador vive lo más que pueda con las personas, y solo lo será en la medida en que sea percibido como una "buena persona", franca, honesta, inofensiva y digna de confianza..." (pág. 89). En este sentido, las observaciones realizadas permitirán a la investigadora establecer contacto con los informantes, interactuando con el entorno y usando al mismo tiempo notas de campo, con relación a temas referentes a las actividades lúdicas y la tabla periódica. 
Por otra parte, se aplicará la entrevista en profundidad a los informantes donde se les preguntará a los docentes sobre diferentes tópicos que permitan determinar el nivel de conocimiento que tienen en relación al reciclaje como alternativa de formación en la conservación del medio ambiente, accediendo a expresar de forma libre las opiniones referentes al tema de investigación. Para la planificación de la entrevista se realizarán las preguntas y se clasificarán los temas con anterioridad con el fin de evitar la improvisación y vacíos en la conversación.

Luego de tener la información se procederá a realizar la categorización de los informantes, la cual consistirá en un primer momento en la lectura detallada, el análisis, y la decodificación. Después de haber desarrollado lo antes mencionado se dará el proceso de triangulación de la información tomando como base las categorías por cada informante, teorías resaltantes de la investigación y la interpretación de la investigadora, con la finalidad de poder generar la teorización que es la argumentación final, siendo coherente y lógico integrando todos los resultados obtenidos de la información.

4.2.2. Fase 2. Elaboración del plan acción. En esta fase se diseñará el plan acción, como resultado de la información recogida en el diagnóstico, por consiguiente, el diseño se reflejará por un conjunto de actividades que servirán para desarrollar las actividades relacionadas con establecer el reciclaje como alternativa de formación en la conservación del medio ambiente en docentes del C.E.I.N. "Sebastián Araujo Briceño". Las actividades diseñadas se realizarán pensando en las necesidades que presentan los docentes, tomando en cuenta la disposición, recursos y tiempos disponibles que le permitieran a los involucrados participar de forma activa en las actividades de incorporación. 4.2.3. Fase 3. Ejecución del plan acción. Consistirá en llevar a cabo la ejecución de las actividades lúdicas bajo una programación estableciendo referente a estrategias, contenido, actividades, día, fecha, recursos, responsables y acciones en los y las docentes del C.E.I.N. "Sebastián Araujo 
Briceño". La aplicación se desarrollará en los entornos de la escuela, propuesta por los involucrados y la investigadora quien será la responsable de llevar a cabo los objetivos propuesto en la investigación acción.

4.2.4. Fase 4. Valoración del plan acción. Permitirá valorar las actividades aplicadas, programadas en el plan acción, donde se describirá el impacto que tuvo las acciones implementadas durante la investigación, bajo el enfoque cualitativo, donde la investigadora expresará las vivencias observadas en la puesta en práctica de cada actividad lúdica aplicada al grupo. Asimismo, se explicará detalle a detalle la experiencia tanto positiva como negativa de las actividades para establecer el reciclaje como alternativa de formación en la conservación del medio ambiente en docentes del C.E.I.N. "Sebastián Araujo Briceño".

\subsection{Informantes}

Dado que la investigación cualitativa busca la interpretación y comprensión de los fenómenos, para lo cual se pone de manifiesto la descripción y análisis de acciones y vivencias de los individuos, Martínez (2012), recomienda que se "requiere trabajar con muestras pequeñas para facilitar una investigación lo más cercana posible a la realidad" (pág. 135). Por eso se habla de participantes o informantes, quien ha de ser una persona capaz de aportar información sobre el elemento de estudio.

Es de acotar que Vera y Ponce (2000), en el proceso investigativo, la identificación de los participantes, generalmente "es una muestra seleccionada, no aleatoria; ya que el investigador procura por una que concierne más a los propósitos de la investigación" (pág. 23). Considerando lo expresado por los mencionados autores, para fines del presente estudio, como informantes participarán tres (03) docentes que laboran en la institución y gozan de reconocida responsabilidad y compromiso en el C.E.I.N. Cada uno 
ha de ofrecer información relevante en cuanto a las unidades de análisis planteadas en el presente estudio.

\subsection{Técnicas e Instrumentos de Recolección de datos}

Las técnicas para la recolección de datos, son los distintos procedimientos, formas o maneras que se emplean al recoger información. Según Vallejo (2011) expresa que "existe variedad de técnicas e instrumentos de recogida de datos de acuerdo a la temática a investigar, los objetivos propuestos, la adecuación a cada situación y su mayor o menor conocimiento" (pág. 150). Es así como, las técnicas de investigación representan un elemento clave para el investigador en el proceso de recolección de la información.

A tales fines, Pérez (2011), manifiesta que, entre las técnicas de recolección de datos en una investigación cualitativa, figuran las "de papel y lápiz, notas de campo, el diario, anécdotas, comentarios, entrevistas y grabaciones entre otras" (pág. 156). Es de agregar que la mencionada autora, expresa que las técnicas de recolección de datos en la investigación cualitativa son diversas; porque se trata de obtener el máximo de información pertinente al estudio, que genere interpretación más aproximada de la realidad y de los factores incidentes en ella. Por tanto, en este caso se utilizará como técnica la entrevista y la observación directa.

En cuanto a las entrevistas las mismas constituyen una estrategia clave para la recopilación de los datos para el estudio que se realiza. De acuerdo a Báez (2011), en la entrevista "el investigador prepara de antemano una lista de preguntas; pero sin limitar la misma a ciertas preguntas, ni fijar previamente el orden en las que se contestan" (pág. 21). Por tanto, su uso permitirá sondear las respuestas y desarrollar temas que surgen en el transcurso de la entrevista, aclarar términos, definir problemas, orientar, motivar al entrevistado, elevar su nivel de interés y colaboración y estimular su memoria. 
Asimismo, Ramírez (2012) expresa que la observación participante "es cuando para obtener los datos el investigador se incluye en el grupo, hecho o fenómeno observado, para conseguir la información desde adentro" (pág. 112). Para la presente investigación, la investigadora actuará desde dentro, pues forma parte del grupo de docentes que forma a los estudiantes que representan el campo de investigación.

En lo que respecta a los instrumentos, Báez (2011), señala que éstos "consisten en formularios diseñados previamente para registrar la información durante el proceso de recolección de datos" (pág. 154). Es de referir que para esta investigación se empleará como instrumento la guía de entrevista para abordar los docentes de aula y una guía de observación para registrar las observaciones hechas.

\subsection{Validez y Fiabilidad de los Instrumentos}

Cabe agregar que la guía de entrevista y guía de observación como instrumentos no serán sometidos a procesos de validación mediante expertos, por cuanto no se trata de cuantificar los hechos, sino más bien comprender mejor la naturaleza del fenómeno, acerca de establecer el reciclaje como alternativa de formación en la conservación del medio ambiente en docentes del C.E.I.N. "Sebastián Araujo Briceño" de Ciudad Bolivia, municipio Pedraza estado Barinas.

En este orden de ideas, puede decirse que para la validación del instrumento que se aplicará será interna, puesto que tal como lo refiere Pérez (2012: 156), se garantizan por la aplicación de procesos holísticos de la investigación, entrevistas; triangulación de las diversas fuentes de datos, permitiendo que todos los involucrados se expresen y aporten variedad de información que no se puede obtener mediante preguntas solamente cerradas.

Al efecto, la mencionada autora agrega, que la validez de los instrumentos "es el grado en que las respuestas son independientes de las 
circunstancias accidentales de la investigación y en la medida de que sea válido lo que se interpreta en forma correcta" (pág. 157). En consecuencia, la validez, se sustenta en la forma cómo se recogerán los datos necesarios para la mejor comprensión del fenómeno estudiado, la captación de los momentos, se analizan e interpretan los acontecimientos dentro de su propio contexto.

Además, es de considerar que tal como lo señala Martínez (2012), en la investigación cualitativa se habla de "fiabilidad de la investigación misma" (pág. 86). Además en opinión del autor, los instrumentos utilizados para recabar información se constituyen en elementos que fortalecen, testimonian y respaldan el estudio realizado. Puesto que la fiabilidad constituye el grado en que las respuestas son independientes de las circunstancias accidentales de la investigación.

De allí, que para garantizar la fiabilidad de la investigación, el mencionado autor, sugiere utilizar categorías descriptivas concretas y precisas, con datos primarios y no manipulados; igualmente, solicitar la colaboración de los informantes para constatar la objetividad de las notas de campo y, hacer uso de instrumentos de investigación que evidencien la realidad estudiada a través de imágenes y sonidos para que pueda ser verificada y analizada posteriormente por otros investigadores o evaluadores de la investigación.

En este particular, la misma, estará dada por el proceso de triangulación de las distintas fuentes de datos (lo observado por el investigador, lo expresado por los profesores, el coordinador pedagógico y el directivo), permitiendo interpretar la situación objeto de estudio a la luz de las evidencias provenientes de los informantes.

\subsection{El Análisis de los Datos}

El análisis de los datos, es un proceso sistemático, coherente que resulta de la organización de la información, con miras a interpretar 
globalmente el fenómeno de estudio, al aplicar los instrumentos de recolección de datos y otros medios o técnicas. De esta manera, tal como lo considera Vera y Ponce (2012), constituye uno de los momentos de mayor síntesis e integración de la información que se obtiene "al aplicar diversos instrumentos y medios de observación, preponderando más, un análisis descriptivo coherente para lograr una interpretación minuciosa y detallada del asunto o problema de investigación" (pág. 33).

Cabe agregar, que en la investigación cualitativa, el análisis de los datos se apoya en la triangulación. Esta, de acuerdo a lo señalado por Báez (2011) constituye "un proceso de verificación" (pág. 15). Agregan además, los autores que este término se usa para describir el procedimiento en el que una parte de la información es respaldada por otras fuentes de datos.

\section{Conclusiones.}

Una vez analizados e interpretados los datos obtenidos en el diagnóstico y tomados como referencia los objetivos y el cúmulo de preguntas orientadas en la investigación, se concluye:

La comunidad en cuanto a los riesgos que se corren con la proliferación de la contaminación ambiental, se pudo constatar que gran parte de la comunidad desconocían los riesgos a los que podría estar expuesto por la incorrecta utilización de los residuos sólidos.

Es importante resaltar que, la comunidad, una vez que recibió los talleres de sensibilización, se mostraron preocupados por la problemática, lo que permite inferir el éxito de la sensibilización en torno a la correcta utilización de los residuos sólidos.

Se lograron las metas establecidas para la reutilización de los residuos sólidos, pues los habitantes a través de las diferentes formaciones en los talleres de sensibilización, se mostraron muy preocupados e interesados en 
colaborar para erradicar la problemática de la mala utilización de los residuos sólidos.

Se determinó la creación de acuerdos de convivencia comunitaria, donde ha de establecerse convenios que permitan sancionar a los vecinos que por desconocimiento o mala intención intenten infringir los acuerdos en relación a la correcta utilización de los residuos sólidos.

\section{Referencias}

Carvajal, P. (2012). Presente y futuro del reciclaje en Colombia. [Documento en línea]. Recuperado de:

http://www.elempaque.com/temas/Presente-y-futuro-del-reciclaje-enColombia+4087864

Gómez (2014). El reciclaje: alternativa ecológica para la humanidad. Artículo arbitrado. Revista Ciencias de la Educación. Segunda etapa, año 2014, volumen 24, № 44. Valencia, Venezuela.

González, M., (2002). La Educación Popular Ambiental. Caracas, Venezuela: Revista Educación, Participación y Ambiente. MARN.

Osorio, M. (2012). Alternativas viables del reciclaje. Editorial Com-Ba. Bogotá, Colombia.

Ramírez, A. (2013). Conservación Ambiental en Venezuela. Revista Educare. Universidad de los Andes, Mérida, Venezuela. Edición № 61. Rodríguez, R. (2014). La formación ambiental del educador. Ediciones CANARIA, Madrid, España.

Martínez (2002). La Nueva Ciencia: su desafío, lógica y método. México: Trillas. 


\section{Francys Yuviana Garrido Rojas}

e-mail: enyuby@hotmail.com

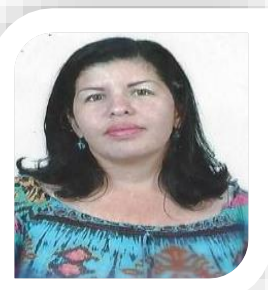

Docente en educación inicial, nacida en Ciudad Bolivia Pedraza estado Barinas Venezuela. Actualmente reside en la misma Ciudad. Realizo estudios de primaria del primero a tercer grado en la Escuela Básica Nacional "Ana de Romero" (Pedraza). culminando la primaria en la Escuela Básica "Sebastián Araujo Briceño" (Pedraza), secundaria del primer año al tercer año en la Escuela Técnica Agropecuaria "Euclides Moro" (Municipio Zamora Estado Barinas) culminando el cuarto y quinto año de Bachiller en Ciencias y Humanidades en el colegio "Nuestra señora de Altagracia" (Pedraza). Egresada del Instituto Universitario de Tecnología "Agustín Codazzi" (Barinas) como técnico superior en Educación Preescolar (2004) y culmina la carrera en la Universidad Pedagógica Experimental Libertador Núcleo Barinas, obteniendo el título de Prof. en Educación Inicial (2008), con Diplomado en orientación de la conducta del ser humano universidad del estado Zulia "LUZ" (2012). En el presente cursando estudio de maestría en Educación Ambiente y Desarrollo en la Universidad Pedagogía Experimental Libertador (UPEL) Barinas. En este momento desempeñando funciones como Docente coordinadora de cultura en el C.E.I.N. "Sebastián Araujo Briceño" Municipio Pedraza Estado Barinas.

El contenido de este manuscrito se difunde bajo una Licencia de Creative Commons ReconocimientoNoComercial-Compartirlgual 4.0 Internacional 